m Jahr 2001 publiziert die Initiative „Allergic Rhinitis and its Impact on Asthma“ (ARIA) erstmals ihre Leitlinie zur Therapie von allergischer Rhinitis und Asthma, 2008 gab es ein Update doch: „Lohnt sich der Zirkus mit den Leitlinien überhaupt?“, fragte Prof. Dr. Ralph Mösges, Köln, mit Blick auf die hohe Selbstmedikationsrate. Er betonte: "Jeder Zweite geht mit Heuschnupfen nicht mehr zum Arzt.“ Die Flut rezeptfreier Heuschnupfenmittel öffnet dem Tür und Tor. In den USA wurden die Folgen des Selbstmanagements bereits 2008 erfasst: Hier sind nur noch $40 \%$ der Patienten komplett unter ärztlicher Kontrolle. Dabei wäre eine differenzierte Therapie der allergischen Rhinitis möglich und wichtig. Denn, so machte Mösges deutlich: Heuschnupfenpräparate ließen sich nicht über einen Kamm scheren. Das gelte sowohl für die Palette der Antihistaminika als auch für die nasalen Glukokortikoide. Sie unterscheiden sich beispielsweise in der Rezeptoraffinität, der Bioverfügbarkeit - und damit in ihrem Einfluss auf das Längenwachstum von Kindern. Auch die Palette der Nebenwirkungen, etwa das Risiko einer Epistaxis, ist nachweislich unterschiedlich hoch.

\section{Nasenspray plus}

Antihistaminikum?

In der Therapie der allergischen Rhinitis kann es durchaus sinnvoll sein, nasale Glukokortikoide und Antihistaminika zu kombinieren, wenn der Patient „Kortison“ nicht ablehnt und die Compliance stimmt. Eine bessere Therapieadhärenz wird üblicherweise durch Fixkombinationen erreicht, wie sie jetzt auch für die allergische Rhinitis untersucht werden: In einer
Phase-III-Studie erwies sich die Fixkombination aus Fluticason und Azelastin in einer neuen Galenik als effektiver und schneller wirksam im Vergleich mit der jeweiligen Monosubstanz.

\section{Ära der rekombinanten Allergene}

Genau vor 100 Jahren wurde die erste Studie publiziert, in der Heuschnupfenpatienten mit einem natürlichen Gräserpollenextrakt im Sinn einer subkutanen allergenspezifischen Immuntherapie behandelt wurden. Die Qualität solcher natürlicher Allergenextrakte wurde durch unterschiedliche Standardisierungsansätze kontinuierlich verbessert. Nun könnte manchen Extrakten schon in zwei bis drei Jahren das „Aus“ drohen. Prof. Dr. Ludger Klimek, Wiesbaden, prognostizierte: Definierte rekombinante Allergene und die gezielte Konzeption von Designerpräparaten „eröffnen heute den Weg in eine neue Ära“. Erste klinische Studien weisen auf eine schnell einsetzende und anhaltende Wirkung hin.

\section{Noch Zukunftsmusik: Biologicals bei atopischer Dermatitis}

Atopische Dermatitis in der Schwangerschaft? Dann ist die topische Therapie mit den klassischen Glukokortikoiden „mit großer Wahrscheinlichkeit harmlos", wertete Prof. Dr. Andreas Wollenberg, München. Es müsse lediglich mit einem etwas geringeren Wachstum der Kinder gerechnet werden. Orale Glukokortikoide sind in der Schwangerschaft dagegen kontraindiziert. Sie gelten allerdings beim atopischen Ekzem ohnehin als obsolet. Als stark wirksame Option nannte Wollenberg die topischen Calcineurin-Inhibitoren und verwies darauf, dass sie nicht mit einem erhöhten Lymphomrisiko assoziiert seien. Noch nicht für die Therapie zugelassen sind Wirkstoffe aus der Gruppe der Biologicals, von denen einige für diese Indikation intensiv untersucht werden.

\section{Wenn Säuglinge giemen ...}

... ist die Prognose günstig, wie Daten der Studie „Avon Longitudinal Study of Parents and Children (ALSPAC) zeigen, bei der 14.000 Neugeborene in den ersten drei Lebensjahren beobachtet wurden. Unkritisch scheint es danach zu sein, wenn die Kinder bis zum 18. Lebensmonat mit dem Giemen beginnen. Bei $90 \%$ der Kinder bestand dann keine Assoziation mit einer Atopie und keine Persistenz der Beschwerden über das sechste Lebensjahr hinaus. Anders bei Kindern, die nach dem 18. Monat mit dem Giemen beginnen. Bei ihnen bestand eine starke Assoziation mit einem Asthma bronchiale im Schulalter.

\section{Asthmakontrolle via Trial and Error}

Wie sollte die Therapie angepasst werden, wenn Asthma bei Kindern auch durch die Gabe von täglich $200 \mu \mathrm{g}$ Fluticason nicht kontrollierbar ist? Die Studie „Best Addon Therapy Giving Effective Responses“ (BADGER) zeigte, dass die Kombination mit einem lang wirksamen Beta-2-Agonisten dann am häufigsten die beste $\mathrm{Op}$ tion ist. Doch, so Prof. Dr. Jürgen Seidenberg, Oldenburg: $30 \%$ der Studienteilnehmer sprachen am besten auf eine erhöhte Glukokortikoiddosis an, weitere $30 \%$ auf die zusätzliche Gabe eines Leukotrienantagonisten. Sein Fazit: Die Patienten reagieren sehr unterschiedlich. Prädiktive Paramater gibt es nicht. Da hilft nur ausprobieren.

Dr. Beate Fessler 